\title{
Pyoints: A Python package for point cloud, voxel and raster processing.
}

\section{Sebastian Lamprecht ${ }^{1}$}

1 Trier University

DOI: $10.21105 /$ joss.00990

\section{Software \\ - Review ¿ \\ - Repository ¿ \\ - Archive ${ }^{\top}$}

Submitted: 27 September 2018 Published: 03 April 2019

\section{License}

Authors of papers retain copyright and release the work under a Creative Commons Attribution 4.0 International License (CC-BY).

\section{Summary}

The evolution of automated systems like autonomous robots and unmanned aerial vehicles leads to manifold opportunities in science, agriculture and industry. Remote sensing devices, like laser scanners and multi-spectral cameras, can be combined with sensor networks to all-embracingly monitor a research object.

The analysis of such big data is based on geoinformatics and remote sensing techniques. Today, next to physically driven approaches, machine learning techniques are often used to extract relevant thematical information from the data sets. Analysis requires a fusion of the data sets, which is made difficult conceptually and technically by different data dimensions, data structures, and various spatial, spectral, and temporal resolutions.

Today, various software to deal with these different data sources is available. Software like GDAL (GDAL/OGR contributors, 2018) and OpenCV (Bradski, 2000) are intended for image processing. Libraries, like PCL (Rusu \& Cousins, 2011), Open3D (Zhou, Park, \& Koltun, 2018) and PDAL (PDAL contributors, 2018) focus on 3D point cloud processing. Each of these software packages provide an API specially designed to solve the problems of their field efficiently. When developing algorithms for automated processing of various types of input data, the differing APIs and programming languages of these software packages become a drawback. To support fast algorithm development and a short familiarization, a unified API would be desirable.

Pyoints is a python package to conveniently process and analyze point cloud data, voxels, and raster images. It is intended to be used to support the development of advanced algorithms for geo-data processing.

The fundamental idea of Pyoints is to overcome the conceptual distinction between point clouds, voxel spaces, and rasters to simplify data analysis and data fusion of variously structured data. Based on the assumption that any geo-object can be represented by a point, a data structure has been designed that provides a unified API for points, voxels, and rasters. Each data structure maintains its characteristic features, to allow for intuitive use, but all data is also considered as a two or three dimensional point cloud, providing spatial indices that are required in many applications to speed up spatial neighborhood queries.

During development, great emphasis was put on designing a powerful but simple API while also providing solutions for most common problems. Pyoints implements fundamental functions and some advanced algorithms for point cloud, voxel, and raster data processing, like coordinate transformation, vector algebra, point filters, and interpolation. Pyoints also provides a unified API for loading and saving commonly used geo-data formats.

Pyoints was designed to support research activities and algorithm development in the field of geoinformatics and remote sensing. Early versions of the software have been 
used for some pre-studies at Trier University (Lamprecht, Hill, Stoffels, \& Udelhoven, 2017; Lamprecht, Stoffels, Dotzler, Haß, \& Udelhoven, 2015). Pyoints is also used in the PANTHEON project (PANTHEON consortium, 2018) to monitor hazelnut orchards.

The source code of Pyoints is on GitHub (Lamprecht, 2019a). The documentation can be found on GitHub Pages (Lamprecht, 2019b).

\section{Acknowledgements}

This work has been supported by the European Commission under the grant agreement number 774571 Project PANTHEON.

\section{References}

Bradski, G. (2000). The OpenCV Library. Dr. Dobb's Journal of Software Tools. Retrieved from https://opencv.org

GDAL/OGR contributors. (2018). GDAL/OGR geospatial data abstraction software library. Open Source Geospatial Foundation. Retrieved from http://gdal.org

Lamprecht, S. (2019a). Pyoints. doi:10.5281/zenodo.2557574

Lamprecht, S. (2019b). Pyoints documentation. Retrieved April 1, 2019, from https: //laempy.github.io/pyoints/

Lamprecht, S., Hill, A., Stoffels, J., \& Udelhoven, T. (2017). A Machine Learning Method for Co-Registration and Individual Tree Matching of Forest Inventory and Airborne Laser Scanning Data. Remote Sensing, 9(5), 505. doi:10.3390/rs9050505

Lamprecht, S., Stoffels, J., Dotzler, S., Haß, E., \& Udelhoven, T. (2015). ATrunk-an als-based trunk detection algorithm. Remote Sensing, 7(8), 9975. doi:10.3390/rs70809975

PANTHEON consortium. (2018). Precision farming of hazelnut orchards. Retrieved August 10, 2018, from http://www.project-pantheon.eu/

PDAL contributors. (2018). PDAL: The point data abstraction library. Retrieved from https://pdal.io/

Rusu, R. B., \& Cousins, S. (2011). 3D is here: Point Cloud Library (PCL). IEEE International Conference on Robotics and Automation, 1-4. doi:10.1109/ICRA.2011.5980567

Zhou, Q.-Y., Park, J., \& Koltun, V. (2018). Open3D: A modern library for 3D data processing. arXiv:1801.0984\%. 